\title{
Seasonal Alteration in Amounts of Lignans and Their Glucosides and Gene Expression of the Relevant Biosynthetic Enzymes in the Forsythia suspense Leaf
}

\author{
Kinuyo Morimoto and Honoo Satake* \\ Bioorganic Research Institute, Suntory Foundation for Life Sciences; 1-1-1Wakayamadai, Shimamoto Mishima, \\ Osaka 618-8503, Japan. \\ Received June 4, 2013; accepted July 1, 2013; advance publication released online July 6, 2013
}

\begin{abstract}
Lignans of Forsythia spp. are essential components of various Chinese medicines and health diets. However, the seasonal alteration in lignan amounts and the gene expression profile of lignan-biosynthetic enzymes has yet to be investigated. In this study, we have assessed seasonal alteration in amounts of major lignans, such as pinoresinol, matairesinol, and arctigenin, and examined the gene expression profile of pinoresinol/ lariciresinol reductase (PLR), pinoresinol-glucosylating enzyme (UGT71A18), and secoisolariciresinol dehydrogenase (SIRD) in the leaf of Forsythia suspense from April to November. All of the lignans in the leaf continuously increased from April to June, reached the maximal level in June, and then decreased. Ninety percent of pinoresinol and matairesinol was converted into glucosides, while approximately $50 \%$ of arctigenin was aglycone. PLR was stably expressed from April to August, whereas the PLR expression was not detected from September to November. In contrast, the UGT71A18 expression was found from August to November, but not from April to July. The SIRD expression was prominent from April to May, not detected in June to July, and then increased again from September to November. These expression profiles of the lignan-synthetic enzymes are largely compatible with the alteration in lignan contents. Furthermore, such seasonal lignan profiles are in good agreement with the fact that the Forsythia leaves for Chinese medicinal tea are harvested in June. This is the first report on seasonal alteration in lignans and the relevant biosynthetic enzyme genes in the leaf of Forsythia species.
\end{abstract}

Key words biosynthetic enzyme; Forsythia; lignan; lignan glycoside

Lignans have a variety of clinically and dietarily important biological activities. ${ }^{1-3)}$ Lignan biosynthesis is well studied in Linum, Forsythia and other plants. ${ }^{1-7)}$ These plants are used as Chinese medicine and health diets. ${ }^{7-11)}$ Forsythia, commonly known as golden bell flower, is a perennial woody plant, and consists of a large number of varieties, including $F$. suspensa. Forsythia fruit, leaf and flower abundantly produce various lignans. ${ }^{412)}$ In Forsythia, major lignan biosynthesis pathways have been determined (Fig. 1). Pinoresinol, a basal lignan, is stepwisely reduced into secoisolariciresinol via lariciresinol by pinoresinol/lariciresinol reductase (PLR) $)^{13,14)}$ or glucosylated by UGT71A18. ${ }^{15)}$ Secoisolariciresinol is converted into matairesinol by secoisolariciresinol dehydrogenase (SIRD). ${ }^{16)}$ Furthermore, matairesinol is glucosylated or consumed for the biosynthesis of arctigenin by unidentified enzymes (Fig. 1). Arctigenin is also glucosylated to arctiin by an unidentified glucosyltransferases.

Lignans and their glucosides in Forsythia fruits and leaves exhibit diverse biological activities on mammals. ${ }^{1,3,17-20)}$ Most lignans are uptaken directly, or further metabolized by enteric bacteria to enterolactone and enterodiol, designated as phytoestrogens. ${ }^{21,22)}$ There have been increasing reports on the effects of the dietary lignans on reduction of oxidative injury, post-menopause symptoms, and cancer risks. ${ }^{22-27)}$ Notably, each lignan was found to exhibit both similar and differential bioactivities in mammals, which underscores the dietary and medicinal significance of specific production of a targeted lignan, instead of lignan mixtures. ${ }^{3,17-27)}$ These findings suggest a potential increase in the demands of isolated lignans for the development of health diets, supplements, or

The authors declare no conflict of interest. drugs, and the requirement of efficient acquisition of a lignan from source plants. Thus, elucidation of seasonal profiles in lignan amounts and the biosynthetic enzymes is expected to develop novel regulatory systems for efficient production of target lignans in Forsythia species. In this paper, we present the seasonal profiles of pinoresinol, matairesinol, arctigenin, and their biosynthetic enzyme genes (Fig. 1) in the leaves of F. suspense.

\section{MATERIALS AND METHODS}

Plant Material and Leaf Sampling The F. suspense plants were cultivated in the outdoor garden at Suntory Business Expert Limited in Osaka, Japan. Eight to thirty F. suspense leaves located at the outermost branches of the middle parts of the fully grown plants were sampled every month from April 5 to November 15 (Table 1). The sampled leaves were cut into small pieces and mixed well. The mixed leaf pieces were aliquoted into $0.2 \mathrm{~g}$ and kept at $-80^{\circ} \mathrm{C}$ until use. In addition, the climate conditions for each sampling day are summarized in Supplemental data 1.

Lignan Extraction and HPLC Analysis $0.2 \mathrm{~g}$ of the leaf piece was suspended in $1 \mathrm{~mL}$ of $80 \%$ ethanol and $20 \% 0.1 \mathrm{M}$ sodium phosphate buffer $(\mathrm{pH}$ 4.6) and sonicated twice for $15 \mathrm{~s}$ in $4^{\circ} \mathrm{C}$. The mixture was centrifuged and the supernatant was evaporated in vacuo to $200 \mu \mathrm{L}$. The remaining phase was adjusted to $1 \mathrm{~mL}$ by addition of $0.1 \mathrm{~m}$ sodium phosphate buffer. The $\mathrm{H}_{2} \mathrm{O}$ phase containing the lignan glycoside was digested at $40^{\circ} \mathrm{C}$ overnight with $6 \mathrm{U} \mathrm{mL}^{-1}$ almond $\beta$-glucosidase (Sigma-Aldrich, St. Louis, MO, U.S.A.) in $0.1 \mathrm{~m}$ sodium phosphate buffer ( $\mathrm{pH} 4.6$ ). Lignan samples with or without $\beta$-glucosidase treatment were dissolved again with $50 \%$ aqueous acetoni- 


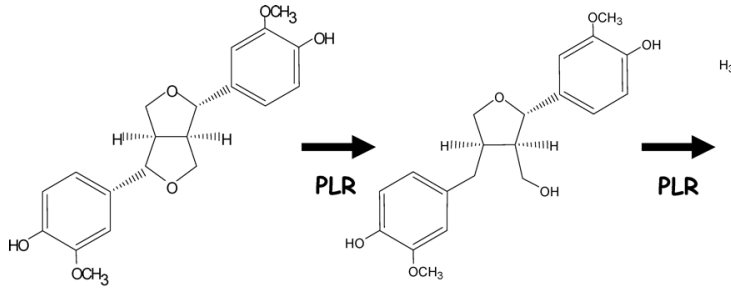

(+)-pinoresinol

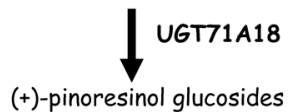

(+)-lariciresinol

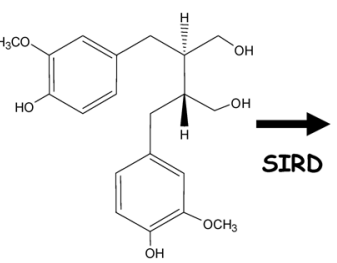

(-)-secoisolariciresinol

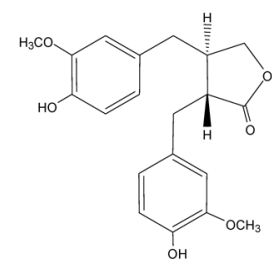

(-)-matairesinol

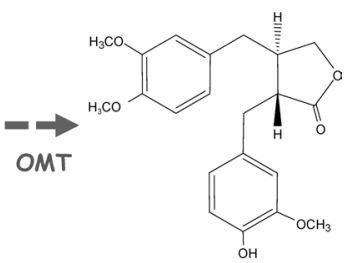

$(-)$-arctigenin
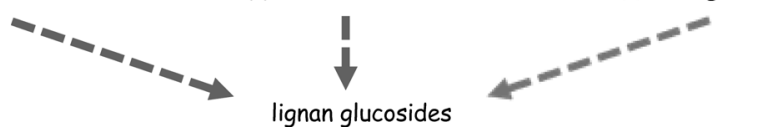

Fig. 1. Lignan Biosynthetic Pathways in Forsythia

PLR, pinoresinol/lariciresinol reductase; SIRD, secoisolariciresinol dehydrogenase; OMT, putative $O$-methyltransferase. Dotted arrows indicate putative steps in Forsythia.

Table 1. Leaf Sample Explanation

\begin{tabular}{|c|c|c|c|c|c|c|c|c|c|}
\hline Sampling date & April 5 & April 15 & May & June & July & August & September & October & November \\
\hline Number of leaves & 16 & 16 & 20 & 25 & 26 & 26 & 30 & 26 & 8 \\
\hline $\operatorname{Min}\left(\mathrm{g}^{-1}\right.$ one leaf) & 0.0001 & 0.0002 & 0.04 & 0.004 & 0.06 & 0.07 & 0.05 & 0.08 & 0.09 \\
\hline $\operatorname{Max}\left(\mathrm{g}^{-1}\right.$ one leaf $)$ & 0.02 & 0.13 & 0.51 & 0.59 & 1.03 & 0.99 & 1.26 & 0.9 & 0.37 \\
\hline $\mathrm{FW}\left(\mathrm{g}^{-1}\right.$ one leaf $)$ & $0.01 \pm 0.009$ & $0.07 \pm 0.048$ & $0.24 \pm 0.151$ & $0.28 \pm 0.215$ & $0.45 \pm 0.279$ & $0.48 \pm 0.295$ & $0.58 \pm 0.393$ & $0.36 \pm 0.26$ & $0.24 \pm 0.096$ \\
\hline DW $(\%)$ & $21.3 \pm 0.45$ & $21.4 \pm 0.17$ & $21.4 \pm 0.18$ & $21.5 \pm 0.18$ & $21.7 \pm 0.31$ & $21.7 \pm 0.30$ & $21.8 \pm 0.19$ & $22.0 \pm 0.12$ & $22.6 \pm 0.11$ \\
\hline
\end{tabular}

The number of leaves on one shoot, minimal and maximal weights of one leaf, and an average of fresh weight (FW) or dry weight (DW).

trile, and analyzed by reverse-phase high performance liquid chromatography (HPLC) using a Develosil C30-UG-5 column (4.6 $\times 150 \mathrm{~mm}$, Nomura Chemical, Aichi, Japan) with an LC2010A HT system (Shimadzu, Kyoto, Japan) as previously reported. ${ }^{28-30)}$ These extraction and HPLC analysis of lignan contents were independently performed three times.

Reverse Transcription Polymerase Chain Reaction (RTPCR) Analyses Total RNA was isolated from the sampled leaf blade using an RNeasy Plant Mini Kit (Qiagen, Valencia, CA, U.S.A.). First-strand cDNA was synthesized using $500 \mathrm{ng}$ of total RNA with a reverse transcriptase SuperScript III (Invitrogen, San Diego, CA, U.S.A.). PCR primer sequences of PLR (accession No. AAC49608), SIRD (accession No. AF352735), UGT71A18 (accession No. AB524718), and ribosome RNA (accession No. AJ236041) are summarized in Supplemental data 2. PCR was performed at $95^{\circ} \mathrm{C}$ for $1 \mathrm{~min}$ followed by 30 cycles at $94^{\circ} \mathrm{C}$ for $20 \mathrm{~s}$, at $52-58^{\circ} \mathrm{C}$ for $30 \mathrm{~s}$, at $72^{\circ} \mathrm{C}$ for $1 \mathrm{~min}$, and a final extension at $72^{\circ} \mathrm{C}$ for $7 \mathrm{~min}$ on a thermal cycler (ABI GeneAmp 9700) using ExTaq DNA polymerase (TaKaRa Bio Inc., Kyoto, Japan). PCR products were resolved on $1 \%$ agarose gel.

Statistical Analysis Results are expressed as means \pm S.E.M. for indicated number of observations. Data were analyzed by one-way ANOVA with Dunnett error protection. Differences were accepted as significant for $p<0.05$.

\section{RESULTS}

Growth of $\boldsymbol{F}$. suspensa under Natural Condition F. suspense leaves generated new buds in early April. On April 5, the buds rapidly developed to 2 to $4-\mathrm{cm}$ length young leaves (Fig. 2). On April 15, the branches developed to $10-\mathrm{cm}$ long, and the leaves gradually further grew to a $5-\mathrm{cm}$ length and $2.5-\mathrm{cm}$ width, and stooped in June. From July to September, no morphological changes in the leaves were observed.
In September, the leaves started to turn yellowish at the leaf edge and gradually turned to brown. The leaves were gradually withered from September to November, and then completely dropped. The average weight of the leaves was rapidly increased from April ( $0.01 \mathrm{~g})$ to September $(0.58 \mathrm{~g})$, but decreased from September, ultimately to $0.24 \mathrm{~g}$ in November (Table 1).

Seasonal Alteration in Lignan Amounts in $F$. suspensa Leaves The major lignans in Forsythia leaves are matairesinol, arctigenin, pinoresinol and their glucosides. ${ }^{1)}$ Total pinoresinol (aglycone and glucosides) amounts were constantly low $[1-1.6 \mathrm{mg} / \mathrm{g}$ of the dry weight of the leaf (DW)], and statistically significant alteration in amounts was not found (Fig. 3A), compared to matairesinol and arctigenin throughout season (Figs. 3B, C). Pinoresinol aglycone was also consistently detected at low level (approximately $0.2 \mathrm{mg} / \mathrm{g} \mathrm{DW}$ ) from April to September, and further decreased from October. Total matairesinol increased from 10 to $20 \mathrm{mg} / \mathrm{g}$ DW for two months as leaves grew (Fig. 3B). The amount of matairesinol peaked in June and then gradually decreased to $2 \mathrm{mg} / \mathrm{g}$ DW in November. The matairesinol aglycone content went from 0.2 to $2.0 \mathrm{mg} / \mathrm{g} \mathrm{DW}$, and the maximal level was observed in July (Fig. 3B). Total arctigenin content was $12.5 \mathrm{mg} / \mathrm{g}$ DW in April 5 and increased to $30 \mathrm{mg} / \mathrm{g} \mathrm{DW}$ in June, and then decreased to $4 \mathrm{mg} / \mathrm{g}$ DW in October (Fig. 3C). These results confirm that matairesinol and arctigenin and their glucosides account for approximately $90 \%$ of all lignans in Forsythia leaves.

The pinoresinol aglycone ratio to total pinoresinol was almost constant until September, and rapidly decreased from October (Fig. 4A). The matairesinol aglycone ratio consistently increased in April 15 (15\%) to November (29\%) except for a slight decrease in May (Fig. 4B). The arctigenin aglycone ratio was basically decreased in April 15 (64\%) to November (24\%) except for a weak (statistically insignificant) increase in May and July (Fig. 4C). It is noteworthy that arctigenin 

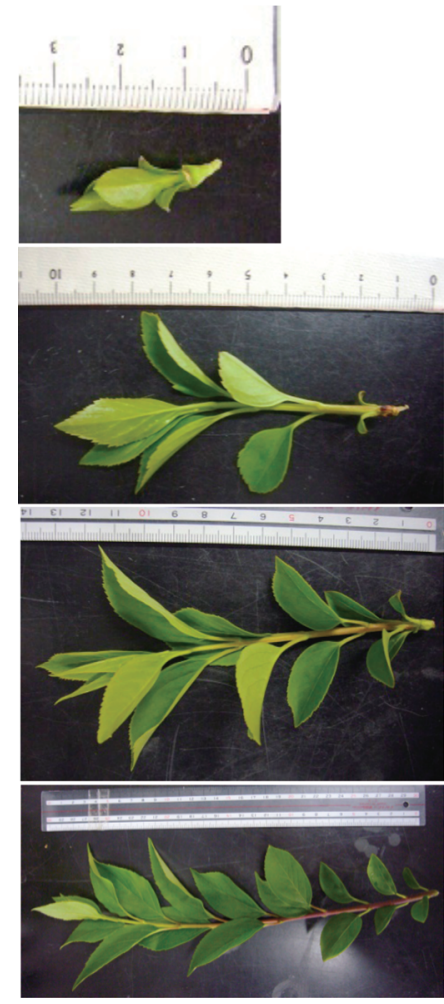

April 15

April 5

May

June

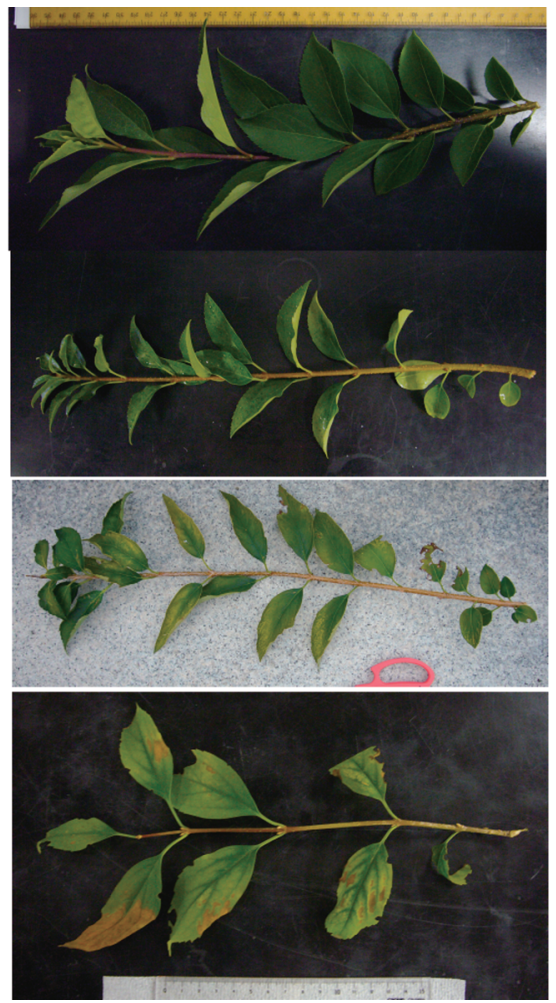

July

September

October

November

Fig. 2. F. suspensa Bud and Leaf from April to November

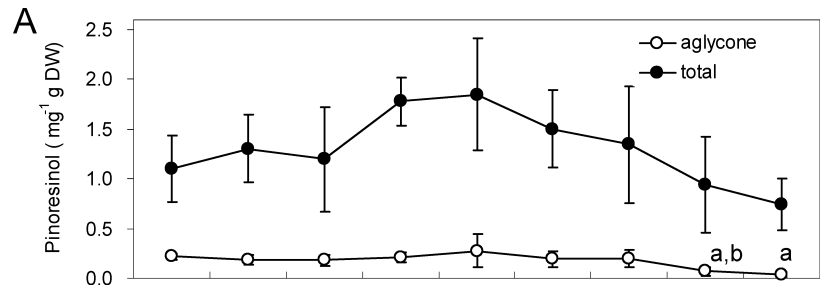

B
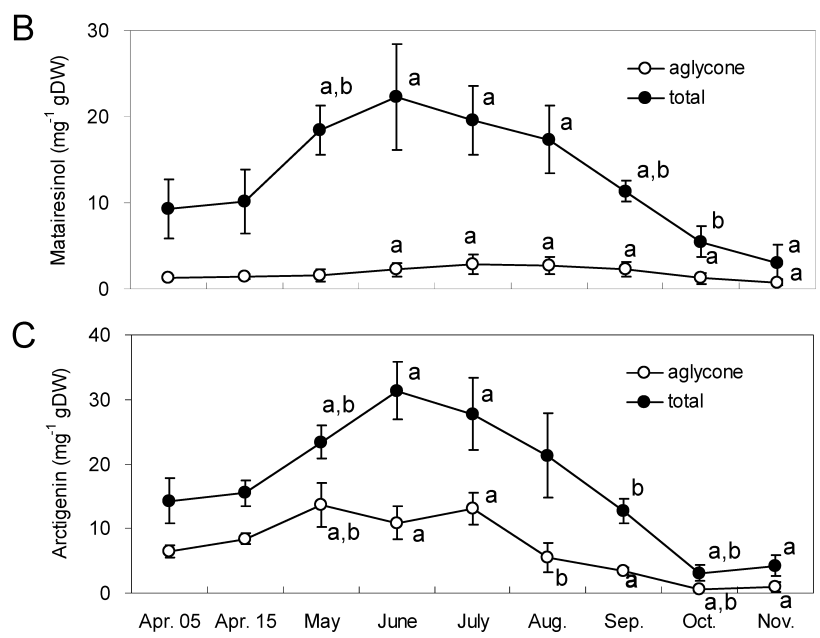

Fig. 3. Lignan Amounts of $F$. suspensa Leaf from April to November

(A) Pinoresinol, (B) Matairesinol, (C) Arctigenin. Open and filled circles indicate aglycone and total lignan (aglycone+glucosides), respectively. All of the lignans were assessed separately for each sample and expressed as the mean \pm S.E.M. of three independent experiments $\left({ }^{a} p<0.05 v s\right.$. April $5,{ }^{b} p<0.05 v s$. each last month).

aglycone is initially produced at high percentage, compared to the aglycones of pinoresinol and matairesinol. These results reveal that each of the three lignan aglycones shows unique

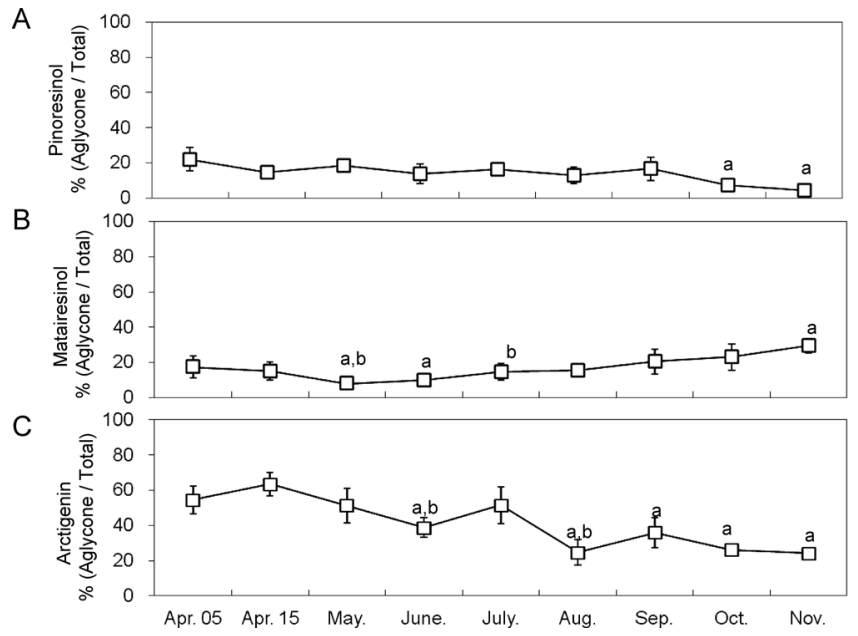

Fig. 4. Percentage of Aglycone/Total of F. suspensa Leaf from April to November

A) Pinoresinol, (B) Matairesinol, (C) Arctigenin. All of the percentages were assessed separately for each sample and expressed as the mean \pm S.E.M. of three independent experiments $\left({ }^{a} p<0.05 v s\right.$. April $5,{ }^{\mathrm{b}} p<0.05 v s$. each last month).

alteration in amounts, and that the arctigenin aglycone ratio most prominently varied of the three lignans throughout the seasons.

Seasonal Gene Expression Alteration in $F$. suspensa Leaf Subsequently, we examined alteration in gene expression of PLR, SIRD, and UGT71A18 (Fig. 1) in F. suspensa leaves from April to November (Fig. 5). PLR was constantly expressed from April to August, whereas no PLR expression was observed from September to November. SIRD showed the variable expression profiles; it was intensely expressed on April 5 and May 15 with a marked reduction on April 15, and 


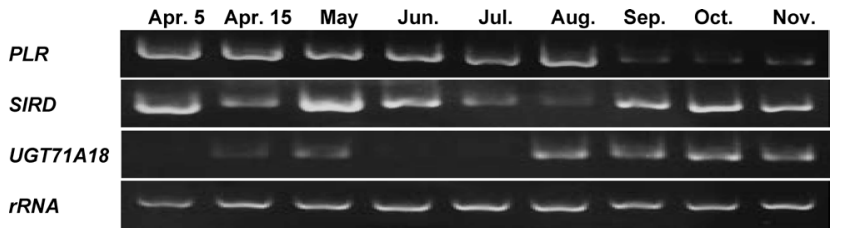

Fig. 5. Gene Expression of Lignan Biosynthetic Enzymes in the Forsythia suspensa Leaves from April to November

decreased from May to August, whereas the expression was restored in September and the expression level was consistent until November. No or markedly low expression of UGT71A18 was detected from April to July, while stable and clear expression of UGT71A18 was detected from August to November. These results indicate the specific gene expression profiles of $P L R, S I R D$, and UGT71A18.

\section{DISCUSSION}

We originally investigate seasonal alteration in the amounts of lignan aglycone and glucosides and gene expression of lignan-biosynthetic enzymes in $F$. suspensa leaves from April to November, when the leaves were still on the branches. Pinoresinol, matairesinol, arctigenin, and their glucosides were found to be produced at the maximal level from June to July (Fig. 3), when the leaves have fully grown (Fig. 2 and Table 1). Such maximal production of these lignans is in good agreement with the fact that Forsythia leaves for Chinese medicine, health diets and tea are harvested from June to July, given that these lignans are major pharmacologically active components. $^{19-22)}$

Pinoresinol aglycone and its glucosides were detected at a level $(1-1.6 \mathrm{mg} / \mathrm{g}$ DW) considerably less than matairesinol and arctigenin plus their glucosides (Fig. 3). Pinoresinol is a specific substrate of PLR (Fig. 1). Moreover, PLR is highly expressed from April to August (Fig. 5). These findings prove that a large part of pinoresinol is consumed for metabolism by PLR from April to August, which is consistent with the high levels of matairesinol and arctigenin during this period (Fig. 3) and with our previous study demonstrating that RNA interference against PLR in Forsythia leaf suspension culture cells resulted in a 20 -fold greater accumulation of pinoresinol, compared with the intact cells. ${ }^{28)}$ In contrast, $P L R$ is not expressed from September to November, while UGT71A18 is highly expressed (Fig. 5). These lignan amount and gene expression profiles indicate that the pinoresinol metabolism is switched from the PLR-dependent lignan biosynthesis to UGT71A18dependent glucosylation, leading to the remarkable reduction of major lignan after August.

Total matairesinol increased approximately 2-fold in June, compared to April (Fig. 3), which is in good agreement with intense expression of SIRD during this period (Fig. 5). Total matairesinol was still detected at high levels in July and August without statistically significant aglycone percentage (Figs. 3B, 4B), whereas the SIRD expression gradually decreases during this period (Fig. 5). These data suggest the relatively long stock period of matairesinol glucosides. In contrast, the SIRD expression is upregulated again (Fig. 5), whereas total matairesinol rapidly decreases after August (Fig. 3B). These phenomena are interpreted in two ways. First, matairesinol may be more predominantly consumed for the downstream lignan biosynthesis than newly biosynthesized by SIRD. However, this presumption is highly unlikely, given that arctigenin is also rapidly decreased proportionately to matairesinol (Fig. 3C). Second, newly biosynthesized matairesinol is consumed for some physiological functions specific to this period, although no physiological roles of matairesinol in plants have ever been clarified. Nevertheless, several lignans such as lariciresinol and secoisolariciresinol were found to inhibit germination, seedling, or growth, ${ }^{31,32)}$ implying that matairesinol produced from September to November is involved in the suppression of the leaf growth of Forsythia or seedling of other plants around Forsythia.

Unlike pinoresinol or matairesinol, arctigenin aglycone is present at high level as a major final lignan product from April to July (Fig. 4). Moreover, arctigenin is most abundantly contained in Forsythia leaves through the season. In addition, Forsythia suspension culture cells fail to produce arctigenin at a detectable level. ${ }^{28)}$ In combination, these results suggest specific biological roles of arctigenin Forsythia. Unfortunately, neither an arctigenin biosynthetic enzyme nor an arctigeninglucosylating enzyme has so far been isolated in a plant. Molecular and functional characterization of these enzymes, combined with the procedure for generation of transgenic Forsythia ${ }^{33)}$ is expected to provide a crucial clue to understanding the physiological roles of arctigenin.

All of the lignans investigated in this study were accumulated at the high levels from June to August (Figs. 3, 4), when the leaves were also markedly grown (Fig. 2 and Table 1). These results suggest some potential biological roles of lignans in growth or survival of the leaves. To date, no biological roles of lignans in the enhancement of leaf growth have ever been reported. Instead, some lignans including pinoresinol have been shown to exhibit pyrethrum-synergistic or antifeedant effects on insects, ${ }^{3)}$ supporting the view that the lignans abundantly produced during leaf growth participate in defense against defoliators, and thus, assist the growth or survival of the leaves.

Recently, sustainable production of plant secondary metabolites including lignans using plants in closed factories, which is free from climate change risks, has received increasing interest. ${ }^{3)}$ The present data are also expected to contribute to the elucidation of the optimal conditions for efficient production of each lignan by Forsythia in closed plant factories. Such studies are currently in progress.

Acknowledgments We are grateful to Prof. Makoto Suematsu (Keio University and Suntory Foundation for Life Sciences) for fruitful comments on preparation of the manuscript. This work was supported by the Plant Factory Project of the Ministry of Economy, Technology, and Industry, Japan

\section{REFERENCES}

1) Umezawa T. Diversity in lignan biosynthesis. Phytochem. Rev., 2 , 371-390 (2003).

2) Suzuki S, Umezawa T. Biosynthesis of lignans and norlignans. $J$. Wood Sci., 53, 273-284 (2007).

3) Satake H, Ono E, Murata J. Recent advances in metabolic engineering of lignan biosynthesis pathways for the production of transgenic plant-based foods and supplements. J. Agric. Food Chem., 130610120410007, in press (2013). 
4) Tokar M, Klimek B. Isolation and identification of biologically active compounds from Forsythia viridissima flowers. Acta Poloniae Pharmaceutica-Drug Res., 6, 191-197 (2004).

5) Hemmati S, Schmidt TJ, Fuss E. (+)-Pinoresinol/(-)-lariciresinol reductase from Linum perenne Himmelszelt involved in the biosynthesis of justicidin B. FEBS Lett., 581, 603-610 (2007).

6) Bayindir U, Alfermann W, Fuss E. Hinokinin biosynthesis in Linum corymbulosum Reichenb. Plant J., 55, 810-820 (2008).

7) Berim A, Ebel R, Schneider B, Petersen M. UDP-glucose: (6-methoxy) podophyllotoxin 7-O-glucosyltransferase from suspension cultures of Linum nodiflorum. Phytochemistry, 69, 374-381 (2008).

8) Deyama T, Nishibe S, Nakazawa Y. Constituents and pharmacological effects of Eucommia and Siberian ginseng. Acta Pharmacol. Sin., 22, 1057-1070 (2001).

9) Milder IEJ, Feskens EJM, Arts ICW, Bueno de Mesquita HB, Hollman $\mathrm{PCH}$, Kromhout D. Intake of the plant lignans secoisolariciresinol, matairesinol, lariciresinol, and pinoresinol in Dutch men and women. J. Nutr., 135, 1202-1207 (2005).

10) Milder IEJ, Feskens EJM, Arts ICW, Bueno de Mesquita HB, Hollman $\mathrm{PCH}$, Kromhout D. Intakes of 4 dietary lignans and causespecific and all-cause mortality in the Zutphen Elderly Study. Am. J. Clin. Nutr., 84, 400-405 (2006).

11) Saarinen NM, Wärri A, Airio M, Smeds A, Mäkelä S. Role of dietary lignans in the reduction of breast cancer risk. Mol. Nutr. Food Res., 51, 857-866 (2007).

12) Zhang Q, Jia C, Xu H, Wang Y, Zhang M, Huo C, Shi A, Yu S. Chemical Constituents of Plants from the Genus Forsythia. MiniReview Org. Chem., 9, 303-318 (2012).

13) Dinkova-Kostova AT, Gang DR, Davin LB, Bedgar DL, Chu A, Lewis NG. (+)-Pinoresinol/(+)-lariciresinol reductase from Forsythia intermedia. Protein purification, cDNA cloning, heterologous expression and comparison to isoflavone reductase. J. Biol. Chem., 271, 29473-29482 (1996).

14) Nakatsubo T, Mizutani M, Suzuki S, Hattori T, Umezawa T. Characterization of Arabidopsis thaliana pinoresinol reductase, a new type of enzyme involved in lignan biosynthesis. J. Biol. Chem., 283, 15550-15557 (2008).

15) Ono E, Kim HJ, Murata J, Morimoto K, Okazawa A, Kobayashi A, Umezawa T, Satake H. Molecular and functional characterization of novel furoruran-class lignan glycosyltransferases from Forsythia. Plant Biotechnol., 27, 317-324 (2010).

16) Xia ZQ, Costa MA, Pelissier HC, Davin LB, Lewis NG. Secoisolariciresinol dehydrogenase purification, cloning and functional expression. Implications for human health protection. J. Biol. Chem., 276, 12614-12623 (2001).

17) Guo H, Liu AH, Ye M, Yang M, Guo DA. Characterization of phenolic compounds in the fruits of Forsythia suspense by high-performance liquid chromatography coupled with electrospray ionization tandem mass spectrometry. Rapid Commun. Mass Spectrom., 21, 715-729 (2007)

18) Piao XL, Jang MH, Cui J, Piao X. Lignans from the fruits of For- sythia suspensa. Bioorg. Med. Chem. Lett., 18, 1980-1984 (2008).

19) Gao JJ, Hattori M. Metabolic activation of lignans to estrogenic and antiestrogenic substances by human intestinal bacteria. J. Trad. Med., 22, 213-221 (2005).

20) Heinonen $S$, Nurmi $T$, Liukkonen $K$, Poutanen $K$, Wähälä $K$, Deyama T, Nishibe S, Adlercreutz H. In vitro metabolism of plant lignans enterolactone and enterodiol. J. Agric. Food Chem., 49, 3178-3186 (2001)

21) Eden JA. Phytoestrogens for menopausal symptoms: a review. $M a-$ turitas, 72, 157-159 (2012).

22) Miller PE, Snyder DC. Phytochemicals and cancer risk: a review of the epidemiological evidence. Nutr. Clin. Pract., 27, 599-612 (2012).

23) Vanharanta M, Voutilainen S, Nurmi T, Kaikkonen J, Roberts LJ, Morrow JD, Adlercreutz H, Salonen JT. Association between low serum enterolactone and increased plasma F2-isoprostanes, a measure of lipid peroxidation. Atherosclerosis, 160, 465-469 (2002).

24) Virk-Baker MK, Nagy TR, Barnes S. Role of phytoestrogens in cancer therapy. Planta Med., 76, 1132-1142 (2010).

25) Lampe JW, Atkinson C, Hullar MA. Assessing exposure to lignans and their metabolites in humans. J. AOAC Int., 89, 1174-1181 (2006).

26) Ward HA, Kuhnle GG. Phytoestrogen consumption and association with breast, prostate and colorectal cancer in EPIC Norfolk. Arch. Biochem. Biophys., 501, 170-175 (2010).

27) Ayella AK, Trick HN, Wang W. Enhancing lignan biosynthesis by over-expressing pinoresinol lariciresinol reductase in transgenic wheat. Mol. Nutr. Food Res., 51, 1518-1526 (2007).

28) Kim HJ, Ono E, Morimoto K, Yamagaki T, Okazawa A, Kobayashi A, Satake H. Metabolic engineering of lignan biosynthesis in Forsythia cell culture. Plant Cell Physiol., 50, 2200-2209 (2009).

29) Morimoto K, Kim HJ, Ono E, Kobayashi A, Okazawa A, Satake H. Effects of light on production of endogenous and exogenous lignans by Forsythia koreana wildtype and transgenic cells. Plant Biotechnol., 28, 331-337 (2011).

30) Ono E, Nakai M, Fukui Y, Tomimori N, Fukuchi-Mizutani M, Saito M, Satake H, Tanaka T, Katsuta M, Umezawa T, Tanaka Y. Formation of two methylenedioxy bridges by a Sesamum CYP81Q protein yielding a furofuran lignan, (+)-sesamin. Proc. Natl. Acad. Sci. U.S.A., 103, 10116-10121 (2006).

31) Cutillo F, D'Abrosca B, DellaGreca M, Fiorentino A, Zarrelli A. Lignans and neolignans from Brassica fruticulosa: effects on seed germination and plant growth. J. Agric. Food Chem., 51, 6165-6172 (2003)

32) Nishiwaki H, Kumamoto M, Shuto Y, Yamauchi S. Stereoselective syntheses of all stereoisomers of lariciresinol and their plant growth inhibitory activities. J. Agric. Food Chem., 59, 13089-13095 (2011).

33) Morimoto K, Ono E, Kim H-J, Okazawa A, Kobayashi A, Satake H. Ono Eiichiro, Kim HJ, Okazawa A, Kobayashi A, Satake H. The construction of transgenic Forsythia plants: comparative study of three Forsythia species. Plant Biotechnol., 28, 273-280 (2011). 